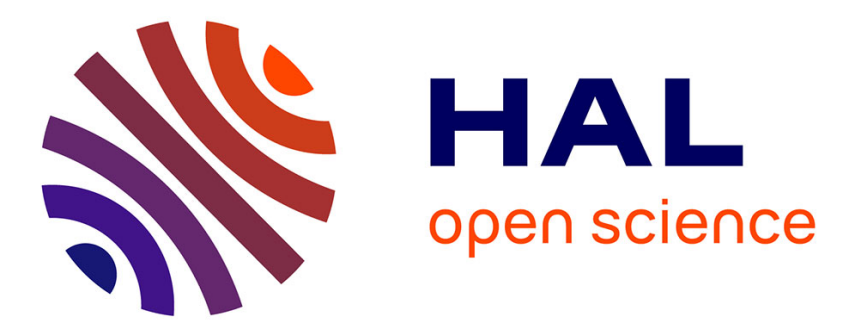

\title{
Nouvelles données chronostratigraphiques concernant les dépôts lacustres de Vialette (Haute-Loire)
}

D. Biquand, C. Cassignol, A. Chambaudet, J. Couthures

\section{To cite this version:}

D. Biquand, C. Cassignol, A. Chambaudet, J. Couthures. Nouvelles données chronostratigraphiques concernant les dépôts lacustres de Vialette (Haute-Loire). Bulletin de l'Association française pour l'étude du quaternaire, 1981, 18 (2), pp.83 - 87. 10.3406/quate.1981.1411 . hal-03413065

\section{HAL Id: hal-03413065 \\ https://hal.science/hal-03413065}

Submitted on 3 Nov 2021

HAL is a multi-disciplinary open access archive for the deposit and dissemination of scientific research documents, whether they are published or not. The documents may come from teaching and research institutions in France or abroad, or from public or private research centers.
L'archive ouverte pluridisciplinaire HAL, est destinée au dépôt et à la diffusion de documents scientifiques de niveau recherche, publiés ou non, émanant des établissements d'enseignement et de recherche français ou étrangers, des laboratoires publics ou privés. 


\section{Nouvelles données chronostratigraphiques concernant les dépôts lacustres de Vialette (Haute-Loire) \\ D. Biquand, C. Cassignol, A. Chambaudet, J. Couthures}

\section{Résumé}

Cherchant à préciser la chronologie du dépôt lacustre de Vialette, gisement fossilifère bien connu du Pliocène du Velay, nous avons mis en œuvre trois méthodes indépendantes dont nous comparons les résultats :

- la méthode utilisant le système radioactif potassium-argon, appliquée à la coulée basaltique sous-jacente au dépôt,

- la méthode « traces de fission », appliquée au dépôt lui-même (âge obtenu : 3,14 $\pm 0,60$ MA),

- la méthode paléomagnétique, également appliquée au dépôt (polarité positive)

\section{Abstract}

In order to establish the geochronology of the lacustrian sediments of Vialette, a wellknown fossiliferous deposit of the Velay Pliocene, three independante methods were employed : the K/Ar method applied to the underlaying basaltic lava flow, the fission track method applied to the sediment itself (age found : $3,14 \pm 0,60 \mathrm{MY}$ ); with the latter we associated a paleomagnetic study (positive polarity).

\section{Citer ce document / Cite this document :}

Biquand D., Cassignol C., Chambaudet A., Couthures J. Nouvelles données chronostratigraphiques concernant les dépôts lacustres de Vialette (Haute-Loire). In: Bulletin de l'Association française pour l'étude du quaternaire, vol. 18, n², 1981. pp. 8387 ;

doi : https://doi.org/10.3406/quate.1981.1411

https://www.persee.fr/doc/quate_0004-5500_1981_num_18_2_1411

Fichier pdf généré le 19/04/2018 


\title{
NOUVELLES DONNÉES \\ CHRONOSTRATIGRAPHIQUES \\ CONCERNANT LES DÉPÔTS LACUSTRES DE VIALETTE (HAUTE-LOIRE)
}

\author{
par D. BIQUAND*, C. CASSIGNOL **, \\ A. CHAMBAUDET ${ }^{* *}$ et J. COUTHURES***
}

\begin{abstract}
RÉSUMÉ
Cherchant à préciser la chronologie du dépôt lacustre de Vialette, gisement fossilifère bien connu du Pliocène du Velay, nous avons mis en œuvre trois méthodes indépendantes dont nous comparons les résultats :

- la méthode utilisant le système radioactif patossium-argon, appliquée à la coulée basaltique sous-jacente au dépôt,

- la méthode "traces de fission ", appliquée au dépót lui-mème (àge obtenu : 3,14 $\pm 0,60$ M.A.),

- la méthode paléomagnétique, également appliquée au dépót (polarité positive).
\end{abstract}

\section{ABSTRACT}

CHRONOSTRATIGRAPHY OF LACUSTRINE DEPOSITS FROM VIALETTE (HAUTE-LOIRE).

In order to establish the geochronology of the lacustrian sediments of Vialette, a wellknown fossiliferous deposit of the Velay Pliocene, three independante methods were employed : the K/Ar method applied to the underlaying basaltic lava flow, the fission track method applied to the sediment itself (age found : $3,14 \pm 0.60 \mathrm{M} . Y$.); with the latter we associated a paleomagnetic study (positive polarity).

\section{INTRODUCTION}

On pensait anciennement que les dépôts lacustres fossilifères de Vialette étaient, soit antérieurs à la coulée du Tarsou (P. Bout, 1960), soit intercalés entre celle-ci et la coulée de Montaigu considérée comme plus récente (Y. Bandet et al., 1978). Une fouille méthodique a fourni récemment l'occasion de réviser la stratigraphie de l'ensemble du site (J. Couthures, 1979). Les coupes dégagées montrent que les dépôts sont supportés directement par l'entablement basaltique provenant du Tarsou et furent constitués à la faveur d'un lac de barrage alimenté à l'Ouest et au Nord par le versant du Mont-Courant. Enfin, le réexamen du contexte géologique conduit à considérer que la coulée de plateau de Montaigu est antérieure à la coulée du Tarsou. En ce qui concerne la faune remarquons que les genres Bos et Equus, dont " l'apparition » à Vialette et Perrier-Etouaires a servi à définir depuis longtemps le début du "quaternaire " en Europe, n'ont pas été retrouvés lors de nos fouilles. Il semblerait donc que les récoltes ont été attribuées à tort à ces deux gisements, et l'ancienne biozonation, qui s'appuyait sur ces localisations est à revoir.

Une étude géochronologique est entreprise par notre groupe pour préciser ces données, porte à la

\footnotetext{
- Laboratoire de Géomagnétisme, Liniversité Pierre et Marie Curie et E.R. 22 du C.N.R.S., 4, avenue de Neptune. 94100 St Maur des Fossès.

* Centre des faibles radioactivites (C.E.A.-C.N.R.S.), Gif/Yvette.

* * Laboratoire de Chimie Physique, 11, rue Pierre et Marie Curie, 75231 Paris, cedex 05.
} 
fois sur les sédiments et les coulées qui leur sont associées. Nous rapportons dant le présent article les études effectuées sur les sédiments et la coulée du Tarsou : - détermination d'un âge radiochronologique pour l'épanchement du Tarsou en utilisant le système radioactif potassium-argon; - évaluation, à l'aide de la méthode utilisant les traces de fission de l'uranium 238, de l'âge des horizons pyroclastiques associés à la faune; - détermination de la polarité magnétique d'horizons argileux également associés à la faune.

\section{DATATION PAR LA MÉTHODE POTASSIUM ARGON}

Nous avons repris l'étude de la coulée basanitique du Tarsou par K-Ar. Le travail présenté est une variante de la méthode (Bandet et al. 1978) qui n'utilise aucun traceur ou "spike" d'argon 38. L'avantage de cette variante est dans ce cas particu- lier de mesurer l'argon 38 des minéraux et ainsi de pouvoir décider si le prédégazage a été accompagné ou non d'une discrimination isotopique modifiant l'âge apparent (Gourinard, 1975). Les caractéristiques à l'affleurement de la coulée autorisent quelques craintes à ce propos.

Les dosages d'argon ont été effectués par spectroscopie de masse sur mésostase triée par densité (mélange de diidométhane et de benzène). Deux séries de mesures (voir tableau I) ont été faites en prédégazant les échantillons respectivement à l'ambiante et à $300^{\circ} \mathrm{C}$, pendant 12 heures. Dans les deux cas, les isotopes rares 36 et 38 sont dans le même rapport, qui est aussi celui de l'argon de l'air. Il n'y a donc pas de discrimination isotopique sensible au dégazage. L'influence sur l'âge apparent de la température de dégazage ne peut s'expliquer que par un départ d'argon radiogénique. Les âges maximaux donnés ne pourraient donc être considérés que comme des limites inférieures. Par ailleurs, l'altération par lessivage rendant plausible une perte sensible de potassium. Les mesures ne sont pas significatives.

Tabl. I. - Conditions expérimentales et résultats par K/AV pour la coulée du Tarsou

Tabl. I : Experimental conditions and results from the $K / A r$ method for the Tarsou lava flow

\begin{tabular}{lcccccc}
\hline & Masse $(\mathrm{Gr})$ & $\% \mathrm{~K}$ & A total $\times 10^{13}$ & $\mathrm{~A}^{+} / \mathrm{Gr}$ & Age (M.A.) & Précision \\
\hline ambiante & 5,4 & & 4,548 & $2,310.10^{12}$ & 2,91 & $\pm 0,05$ \\
$300^{\circ} \mathrm{C}$ & & 0,76 & 1,440 & $2,057.10^{12}$ & 2,59 & $\pm 0,04$ \\
\hline
\end{tabular}

\section{DATATION PAR LA MÉTHODE DES TRACES DE FISSION}

La méthode des traces de fission, basée sur l'enregistrement des produits de fission de l'uranium 238 ne peut donner dans le cas de Vialette que des résultats entachés d'une erreur statistique importante; en effet, la densité des traces fossiles est faible en raison de l'âge "jeune " des échantillons et de leur faible teneur en uranium. Dans le cortège des minéraux lourds présents dans les dépôts lacustres non remaniés, la phase minérale choisie est le sphène provenant de nuées ardentes, d'origine montdorienne (A. Chambaudet et J. Couthures, 1981). A Vialette, augites aciculaires et quartz éruptifs sont en effet absents des dépôts argileux (beidellite), quant aux apatites biogéniques, provenant de la désagrégation de dents, elles sont trop peu abondantes. Une étude approfondie a été effectuée à partir des sphènes in situ de la section $\mathrm{V}$ des fouilles. La granulométrie des grains était de
$0,2 \mathrm{~mm}$. La révélation complète des traces est obtenue par l'acide chlorhydrique $\left(3 \mathrm{~h} 15^{\prime}-9^{\circ} \mathrm{C}\right)$. Les résultats sont résumés dans le tableau II.

Malgré un flux neutronique choisi pour obtenir une densité importante de traces induites, l'imprécision sur l'âge trace de fission reste notable.

\section{DÉTERMINATION DE LA POLARITÉ MAGNÉTIQUE}

Nous n'avons pu soumettre à l'examen paléomagnétique qu'un petit nombre d'échantillons en raison de la difficulté à trouver des horizons à granulométrie fine sans perturbation mécanique ni altération diagénétique et dont la cohésion mécanique soit suffisante pour permettre les prélèvements. Cinq blocs orientés ont été prélevés dans une des coupes de la section $\mathrm{V}$ des fouilles. Nous avons extrait de ces blocs 12 échantillons cubiques, ayant 
Tabl. II. - Âge obtenu par la méthode des traces de fission de l'uranium appliquée aux horizons pyroclastiques du gisement de Vialette

Table II : Fission track results obtained on the ash-fall of the Vialette deposit

\begin{tabular}{|c|c|c|c|c|c|}
\hline \multirow{2}{*}{$\begin{array}{l}\text { Concentration en } \\
\text { Uranium (ppm) }\end{array}$} & \multicolumn{2}{|c|}{ Nombre de traces comptées } & \multirow{2}{*}{$\begin{array}{l}\text { Rapport } \\
\text { DF/DI }\end{array}$} & \multirow{2}{*}{$\begin{array}{l}\text { Age }^{*} \\
\text { (M.A.) }\end{array}$} & \multirow{2}{*}{$\begin{array}{c}\text { Précision sur } \\
\text { l'âge }^{* *}\end{array}$} \\
\hline & fossiles & induites & & & \\
\hline $2,35 \pm 0,04$ & $\begin{array}{c}27 \\
\text { (pour } 177 \text { cristaux) }\end{array}$ & $\begin{array}{c}1665 \\
\text { (pour } 177 \text { cristaux) }\end{array}$ & 0,016 & 3,139 & $\pm 0,60$ \\
\hline
\end{tabular}

* constante de fission de l'uranium 238 utilisée : 7,03 $10^{-17}$ ans $^{-1}$ (Roberts et al., 1968)

** précision déterminée par une statistique de Poisson

Tabl. III. - Directions moyennes d'aimantation obtenues au cours de l'analyse thermique dans les sédiments de l'une des coupes de la section $\mathrm{V}$ des fouilles du gisement de Vialette

Tabl. III. - Average magnetization direction obtained by thermal analysis of the sediments (section $V$ of the Vialette deposit)

\begin{tabular}{|c|c|c|c|}
\hline & $D$ & $I$ & $\alpha 95$ \\
\hline APRES SEJOUR EN CHAMP NUL & $6,6^{\circ} \mathrm{w}$ & $55,7^{\circ}$ & $7,1^{\circ}$ \\
\hline APRES $110^{\circ} \mathrm{C}$ & $4,5^{\circ} \mathrm{w}$ & $57,5^{\circ}$ & $7,1^{\circ}$ \\
\hline ATTAQUE $218^{\circ} \mathrm{C}$ & $6,6^{\circ} \mathrm{w}$ & $57,6^{\circ}$ & $6,5^{\circ}$ \\
\hline THERMIQUE $310^{\circ} \mathrm{C}$ & $22,5^{\circ} \mathrm{w}$ & $45,4^{\circ}$ & $5,8^{\circ}$ \\
\hline $\begin{array}{l}\text { D : déclinaison moyenne } \\
\text { I : inclinaison moyenne }\end{array}$ & \multicolumn{3}{|c|}{$\begin{array}{l}D: \text { mean declinatio } \\
I: \text { mean inclination }\end{array}$} \\
\hline$\alpha 95$ : demi-angle au sommet du có & \multicolumn{3}{|c|}{$\begin{array}{l}\text { 1: mean inclinatio } \\
\text { à } 95 \% \text { (statistique }\end{array}$} \\
\hline
\end{tabular}

chacun un volume de quelques centaines de $\mathrm{cm}^{3}$, et nous avons effectué la mesure de leur aimantation rémanente à l'aide d'un magnétomètre astatique symétrique. Les roches sédimentaires sont souvent porteuses non seulement d'une aimantation primaire dont la polarité est caractéristique de l'époque du dépôt, mais également d'aimantations secondaires dont la plus fréquente est l'aimantation visqueuse. Celle-ci est une aimantation, parfois très intense, acquise progressivement au cours du temps. Sa polarité actuelle est toujours positive. Pour détruire cette aimantation visqueuse et retrouver l'aimantation primaire, avec sa polarité caractéristique, nous avons effectué les opérations suivantes :

- première mesure effectuée après que les échantillons aient séjourné pendant quelques semaines en champ terrestre compensé;

- desaimantation thermique progressive jusqu'à $310^{\circ} \mathrm{C}$. Selon les évaluations théoriques que l'on peut effectuer à partir de la théorie de Néel, il est certain qu'à cette température l'aimantation visqueuse est totalement éliminee.

La dispersion d'ensemble, à chaque stade de l'analyse, des directions individuelles d'aimanta- tion, est suffisamment modérée pour permettre le calcul de directions moyennes (figure 1 et tableau II). L'aimantation stable, obtenue en fin d'analyse, est de polarité positive. La direction moyenne n'évolue de manière significative qu'entre $218^{\circ} \mathrm{C}$ et $310^{\circ} \mathrm{C}$. Cette évolution est sans doute un arte fact (modifications minéralogiques ?) et ne doit pas être prise en compte.

En faisant l'hypothèse, plausible pour ce sédiment à magnétite non transformé, que l'aimantation stable est une aimantation primaire, acquise au moment de la genèse initiale du dépôt, on est amené à conclure que celui-ci s'est effectué au cours d'une période de polarité directe du champ géomagnétique.

\section{CONCLUSION}

Nous avons vu que la datation par la méthode potassium-argon n'est pas utilisable dans ce cas. Celle utilisant les traces de fission de l'uranium est fiable mais malheureusement le domaine d'incertitude est important. Son résultat est compatible avec 


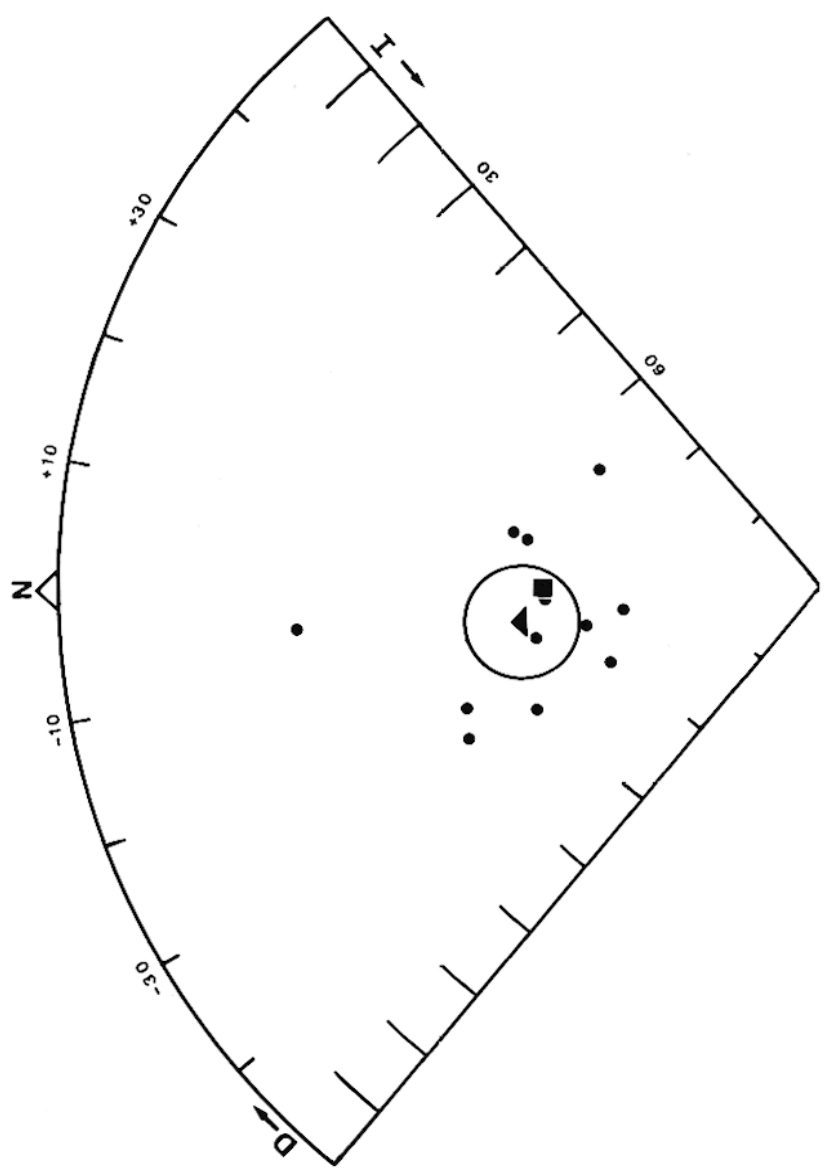

Fig. 1. - Résultats paléomagnétiques obtenus dans l'une des coupes de la Section $\mathbf{V}$ des fouilles du gisement de Vialette. Les directions d'aimantation sont représentées par des points, selon une projection à surfaces conservées.

CERCLES PLEINS : directions d'aimantation correspondant aux différents échantillons analysés, après une attaque thermique à $218^{\circ} \mathrm{C}$

TRIANGLE : direction moyenne, pour la même température. Le cercle représente, selon, la statistique de Fisher, le cône de confiance à $95 \%$ correspondant.

$C A R R E$ : direction du champ dipole axial actuel

Fig. 1. - Paleomagnatic results associated with table III

la polarité magnétique positive. Ces deux résultats considérés simultanément indiquent que le dépôt s'est effectué, au cours de la période de Gauss, à l'exclusion des intervalles de temps représentés par les événements Kaena et Mammoth. La connaissance de la polarité magnétique permet donc de réduire notablement la marge d'incertitude de la datation absolue (figure 2). On mesure bien sur cet exemple l'intérêt de coupler plusieurs techniques. En effet, la méthode paléomagnétique qui seule ne peut avoir qu'un intérêt limité, est par contre un complément très important des datations absolues (traces de fission par exemple) si l'on se trouve à une époque favorable où l'échelle des polarités n'est pas monotone.

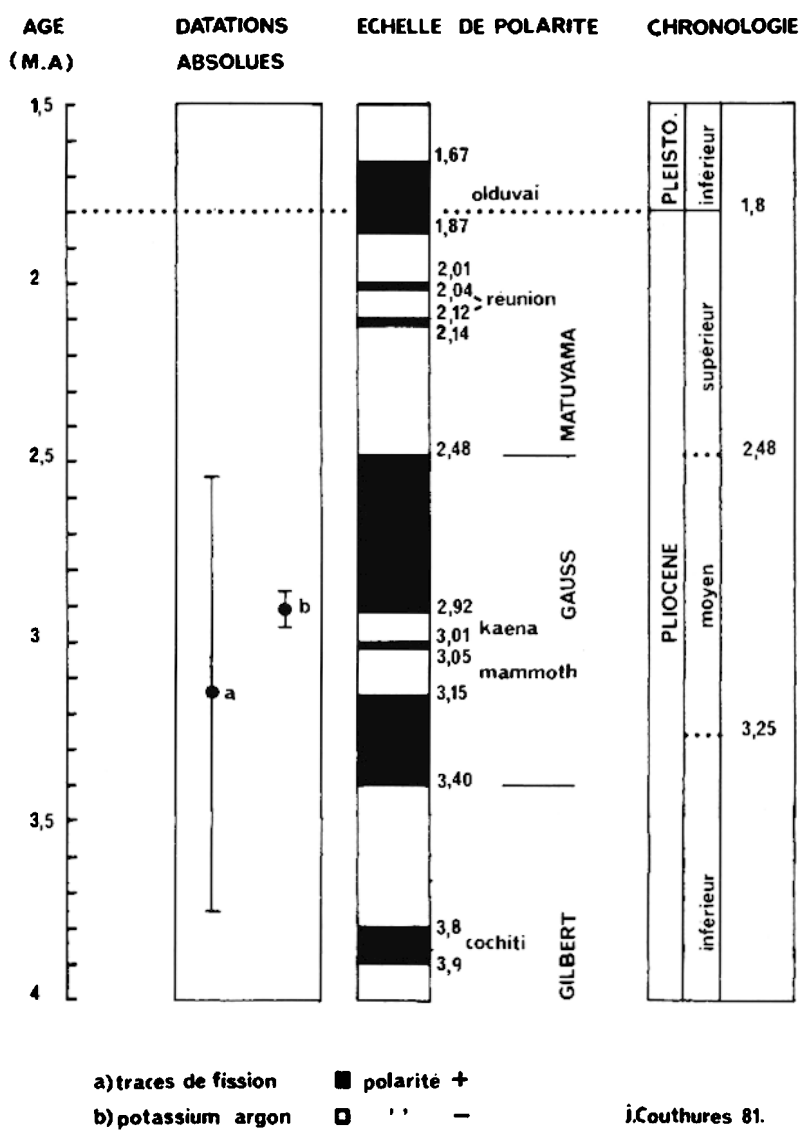

Fig. 2. - Les résultats obtenus à Vialette, replacés dans un cadre géochronologique général, la limite du Pliocène supérieur est celle recommandée par les Congrès tenus Londres en 1948 et Christchruch en 1973. Les limites du Pliocène moyen sont conformes à Couthures J. et al. (1981); l'échelle des polarités est tirée de Mankinen et al. (1979)

Fig. 2. - Fission track and paleomagnetic results in regard to the geochronology time scales

\section{BIBLIOGRAPHIE}

Bandet Y., Donville B. et Michaux J., 1978. Étude géologique et géochronologique du site villafranchien de Vialette (Puy-de-Dôme). Bull. Soc. Géol. France, t. XX, no 3, p. 245-251.

Bout P., 1960. - Le Villafanchien du Velay et du bassin hydrographique moyen et supérieur de l'Allier, Thèse d'État, imp. Jeanne d'Arc, Le Puy, $332 \mathrm{p}$.

Cassignol C., 1978. - Technologie potassium-argon, Rapport CEA, R.4908, division de la physique, Centre de Saclay, 35 p. 
Cassignol C., 1979. - Quelques recherches sur la méthode potassium-argon. Bull. Ass. Fr. Et. Quat., no 58-59 (1-2), p. 27-33.

Chambaudet A. et Couthures J., 1981. - Datations par traces de fission de gisements pliopléistocènes de la périphérie des Mont-Dore (Massif Central, France). C. R. Acad. Sc. Paris, sér. II, t. 293, p. 67-72.

Couthures J., 1979. La stratigraphie du gisement plio-pléistocène de Vialette (Haute-Loire). Bull. Ass. Fr. Et. Quat., 4, p. 171-173.
Couthures J., Biquand D., Chambaudet A. et Cassignol C., 1981. 8 Chronostratigraphie du pliopléistocène d'Auvergne, apports récents du paléomagnétisme et des datations absolues. (A paraitre).

Mankinen E. and Dalrymple B., 1979. - Revised geomagnetic polarity time scale for the interval 0-5 m.y. B.P., Journal of Geophysical Research. Vol. 84, No. B2, p. 615-626.

Roberts J.H., Gad R. and Armani R.J., 1968. Spontaneous fission decay constant of $238 \mathrm{U}$, Phys. Rev., 174, p. 1482-1484. 